\title{
Effect of audio and visual distraction on patients undergoing colonoscopy: a randomized controlled study
}

Authors

Institutions
A. P. De Silva' ${ }^{1}$ M. A. Niriella' ${ }^{1}$, Y. Nandamuni ${ }^{1}$, S. D. Nanayakkara' ${ }^{1}$, K. R. P. Perera² ${ }^{2}$ S. K. Kodisinghe ${ }^{2}$, K. C. E. Subasinghe ${ }^{2}$, A. Pathmeswaran ${ }^{1}$, H. J. de Silva ${ }^{1}$

${ }^{1}$ Faculty of Medicine, University of Kelaniya, Ragama, Sri Lanka

${ }^{2}$ University Medical Unit, Colombo North Teaching Hospital, Ragama, Sri Lanka
Submitted: 25. April 2016 accepted after revision: 9 . September 2016

\section{Bibliography}

Dol http://dx.doi.org/ 10.1055/s-0042-117630 Published online: 20.10 .2016 Endoscopy International Open 2016; 04: E1211-E1214

(c) Georg Thieme Verlag KG Stuttgart · New York E-ISSN 2196-9736

\section{Corresponding author} Professor Arjuna P. De Silva Department of Medicine Faculty of Medicine University of Kelaniya PO Box 6

Thalagolla Road

Ragama

GQ 11010

Sri Lanka

Fax: +94-11-2958337

apdsilva@yahoo.com
Background and study aims: Colonoscopy can cause anxiety and discomfort in patients. Sedation and analgesia as premedication can lead to complications in the elderly and those with comorbidities. This has led to an interest in the use of audio-visual distraction during the colonoscopy. We compared the effects of audio (AD) versus visual distraction (VD) in reducing discomfort and the need for sedation during colonoscopy.

Patients and methods: Consecutive patients undergoing colonoscopy were randomized into three groups: one group was allowed to listen to the music of their choice (AD), the second group was allowed to watch a movie of their choice (VD), and the third group was not allowed either distraction during colonoscopy and acted as a control (C). Patient controlled analgesia and sedation were administered to all three groups. We used $25 \mathrm{mg}$ of pethidine in 5-mg aliquots and $2.5 \mathrm{mg}$ of midazolam in $0.5-\mathrm{mg}$ aliquots. All patients were

\section{Introduction \\ $\nabla$}

Colonoscopy is a relatively safe and effective means of visual inspection of the entire large bowel. It allows diagnostic as well as therapeutic interventions and is the most suitable tool for prevention of colonic malignancies, diagnosis of inflammatory bowel diseases and other structural lesions of the large bowel. However, colonoscopy requires sedation and analgesia during the procedure to minimize patient anxiety and discomfort. Interventions to reduce pre-procedure anxiety and discomfort during the procedure have been identified as the most effective means of improving patient satisfaction of colonoscopy [1]. Patient anxiety before and during the procedure has been shown to affect patient perceived pain, patient cooperation during the procedure, and willingness to undergo repeat colonoscopy [2]. Therefore, some form of sedation and analgesia is administered to patients undergoing colo- assessed for perceived pain and willingness to repeat the procedure. Number of "top-ups" of sedation and total dose of pethidine and midazolam were noted. Patient cooperation and ease of procedure were assessed by the colonoscopist.

Results: In total, 200 patients were recruited [AD, $\mathrm{n}=66$ ( 32 males, median age 57 years); VD, $\mathrm{n}=67$ (43 males, median age 58 years); C, $n=67$ (35 males, median age 59 years)]. The AD group had significantly less pain $(P=0.001)$, better patient cooperation $(P=0.001)$ and willingness to undergo a repeat procedure $(P=0.024)$ compared with VD and $C$ groups.

Conclusions: AD reduces pain and discomfort, improves patient cooperation and willingness to undergo a repeat procedure, and seems a useful, simple adjunct to low dose sedation during colonoscopy.

\section{Study registration: SLCTR/2014/031.}

noscopy. However, routine use of a sedative and analgesic medication is associated with complications and increased procedure cost [3]. Heavy sedation and analgesia as premedication can lead to complications, especially in the elderly and those with comorbidities.

The therapeutic use of audio distraction (AD) in the form of relaxation music has been used in a wide range of practices and has been shown to reduce patient anxiety and improve satisfaction in several scenarios such as in ventilated patient care, peri-operative and postoperative pain management and endoscopic procedures [4]. With the use of $\mathrm{AD}$, the ease with which the physician performs the procedure, time duration of the procedure, dose of sedatives and analgesics used, and patient cooperation were improved along with better patient satisfaction [5]. In addition to its anxiolytic effects, music therapy has been shown to reduce heart rate and blood pressure in patients undergoing lower gastrointestinal endoscopy [6]. 
The therapeutic use of visual distraction (VD) has not been studied or applied to such an extent as music therapy in pain management. A significant reduction in pain scores has been reported among patients undergoing burn wound care when virtual reality was used as a non-pharmacological adjunct in pain management [7]. The use of VD together with ADhas been shown to reduce pain scores and the dose of sedative medication required in patients undergoing colonoscopy. Here the combined distraction was achieved with the use of a movie composed of scenic views and classical music [4]. Another study on patients undergoing screening with flexible sigmoidoscopy showed that patients receiving a combination of audio and visual interventions had lower discomfort and anxiety levels, compared with no intervention or audio stimulation alone [8]. In a more recent study, VD alone using silent movies was shown to improve satisfaction in patients undergoing colonoscopy and decreased anxiety and pain during the procedure among patients with high pre-procedural anxiety scores [9]. The use of a multimodal distraction device, which is an interactive device that uses developmentally appropriate distraction stories and games during painful procedures, was shown to be of benefit in reducing pain and anxiety in children undergoing acute medical procedures [10].

The use of movies with sound or songs, chosen by the patient, as a method of distraction while undergoing colonoscopy, has not been studied. Whether this approach reduces patient anxiety better than when using a set of scenic views or classical music chosen by the investigator has yet to be determined. Furthermore, there are conflicting views with regard to the effectiveness of VD alone, with limited research done in order to evaluate the usefulness of VD as a method of pain management and reduction of anxiety in painful procedures compared to AD. Therefore, we conducted the present study to determine if VD with sound when given in addition to sedation, is an effective means of reducing pain perception, patient anxiety and improving cooperation of the patient when undergoing colonoscopy, compared to sedation alone or sedation with $\mathrm{AD}$ in the form of music.

\section{Materials and methods}

$\nabla$

This was an endoscopist blinded randomized control trial. The study was carried out in the Professorial Endoscopy Unit, Colombo North Teaching Hospital, Ragama, Sri Lanka, from May 2014 to May 2015. Consecutive patients with an indication to undergo elective day-case colonoscopy for medical indications were enrolled in the study in the absence of exclusion criteria. The exclusion criteria included visual or hearing impairment, allergies or hypersensitivity to premedication, patients who had had abdominal surgery or colectomy, personal history of anxiety or psychiatric disorder, and pregnancy. Written informed consent was obtained from all subjects before their participation in the study. Using computer generated numbers, patients were randomly assigned to three groups: the $\mathrm{AD}$ group was allowed to listen to the music of their choice during colonoscopy, the VD group was allowed to watch a film of their choice with sound using a SONY head mounted display (HMZ-T3W-H eye trek system; $\bullet$ Fig. 1), and the control group $(\mathrm{C})$ only received standard sedation during the colonoscopy. For VD, patients were supplied with a list of movies from which they chose any one they preferred. In order to match the variety of interests, we provided Sinhala, Hindi and English action, comedy and cartoon films. For AD, a list of songs of the patient's choice was obtained from a list of songs in Sinha-

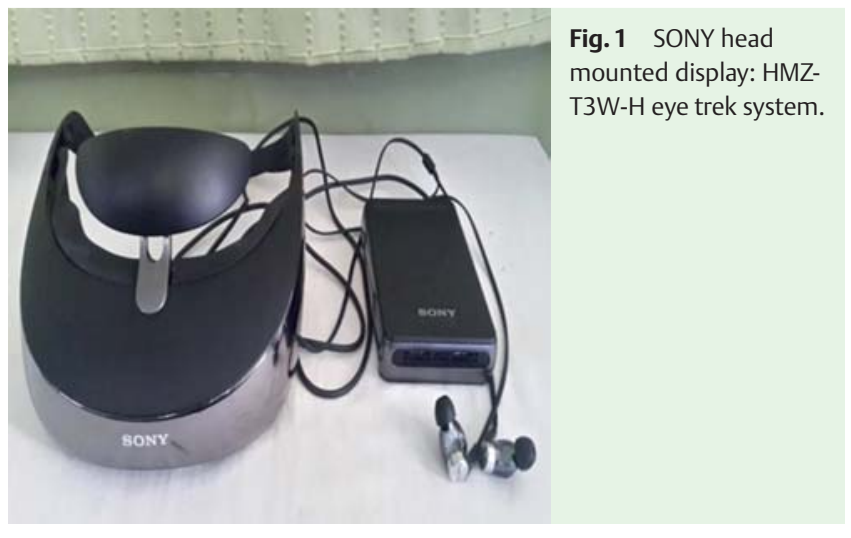

la, Hindi, Classic, and Hip-hop genres. The duration of the movie or video clip and the play list were more than 20 minutes to cover the maximum predicted duration for the colonoscopy. The AD group listened to their choice of music through the SONY head mounted set. The $C$ group had the SONY head mounted set on but with nothing playing for the duration of the procedure. The randomization procedure and the display mounting were performed by doctors who were not endoscopists. This allowed masking of the randomization from the colonoscopist.

All patients were administered midazolam and pethidine for sedation and analgesia, respectively, irrespective of the study group to which they were allocated. The starting dose of midazolam was $1 \mathrm{mg}$ with $0.5-\mathrm{mg}$ increments while that of pethidine was $5 \mathrm{mg}$ with 5-mg increments, until adequate sedation and analgesia was achieved by the patients, respectively. Increments of sedation and analgesia were administered by the endoscopy nurse at the request of the patient. At the end of the procedure, the total doses as well the increments of each drug were recorded.

Oxygen saturation, heart rate, and blood pressure were continuously monitored throughout each colonoscopy. All of the colonoscopies were performed without carbon dioxide insufflation and without the use antispasmodic medication, including butylscopolamine bromide, which could have impacted patient anxiety or pain during colonoscopy. All colonoscopies were performed using Olympus CF-180AL/I colonoscopes (Olympus Corporation, Tokyo, Japan), by senior endoscopists with similar levels of experience and skill.

Primary outcomes assessed were duration of the procedure, doses of analgesics and sedatives required, number of top up doses required, pain and discomfort experienced by the patient during the procedure (using a rating scale), level of cooperation of the patient as evaluated by the physician (using a rating scale) and willingness of the patient to repeat the procedure. Pain perceived by the patient during the procedure was obtained using a visual analog scale, with a score given from 0 to $10(0=$ no pain to $10=$ very painful). The scores were obtained after the patient had fully recovered from the effects of sedation, using an interviewer administered questionnaire. Willingness to undergo repeat colonoscopy if needed ( 1 = never, 2 = maybe/not sure, $3=$ willing) was assessed for each patient. The physician who performed the endoscopy was also given a self-administered questionnaire, using a visual analog scale, with a score ranging from 0 to 10 , to assess the level of cooperation from the patient $(0=$ no cooperation to 10 = very cooperative).

There have been no previous studies directly comparing ADversus VD during colonoscopy. To calculate an approximate sample 
Table 1 Background characteristics of participants.

\begin{tabular}{|llll|}
\hline & Audio distraction (AD) group $(\mathbf{n = 6 6 )}$ & Visual distraction (VD) group (n=67) & Control (C) group (n=67) \\
\hline Males, $n(\%)$ & $32(49)$ & $43(64)$ & $35(52)$ \\
\hline Age, mean (SD), years & $54.4(12.5)$ & $51.5(14.6)$ & $56.5(13.8)$ \\
\hline
\end{tabular}

size, we therefore considered the results of a previous study which compared music versus no music during colonoscopy [4]. We wanted the trial to have a power of $90 \%$ to detect a difference of 1.5 units with an overall significance level of 0.05 . An adjusted significance level of 0.0167 was used for the calculation to account for the comparison of three groups. Therefore, the required sample size for each group was 76. The calculation was performed on Stata version 8.

The analysis was on intention to treat basis. Data were summarized as means and standard deviations or median and interquartile range for the three arms. The Kruskal-Wallis rank test was used to compare the mean scores across the three arms as most of the scores did not have a normal distribution. $P<0.05$ was considered to be statistically significant.

Ethical clearance for the study was obtained from the ethics committee of the Faculty of Medicine, University of Kelaniya. There were no major perceived ethical issues in our study. The trial was registered in the Sri Lanka Clinical Trials Registry (SLCTR), trial registration number 2014/031. The trial was performed in compliance with the Helsinki Declaration and good clinical practice in designing, conducting, recording and reporting of clinical trials. No external funding was obtained to conduct this trial.

\section{Results}

$\nabla$

Two hundred patients were randomized to three groups and the three groups were reasonably similar in terms of age and sex distribution ( $\odot$ Table 1$)$. All randomized patients underwent colonoscopy ( $\bullet$ Fig. 2 ). The cecal intubation rate was $100 \%$ in each group. Total procedure time (SD) in minutes (from setting up of intervention and premedication to recovery from premedication) was similar among the three groups: 25.3 (11.7) for $A D$, 27.4 (12.6) for VD, 27.2 (11.5) for group C, respectively ( $P=0.593$ ). Outcome data are presented in $\bullet$ Table 2 . The ADgroup had statistically significantly less pain, better patient cooperation and was more likely to express a willingness to undergo a repeat procedure. There was no statistically significant difference between the groups in terms of the number of "top-ups" of sedation, total doses of pethidine and midazolam used. Premedication or intervention related adverse effects were not encountered in any group during the study.

\section{Discussion \\ $\nabla$}

The purpose of the present study was to compare the effects of audio (AD) versus visual distraction (VD) in reducing patient discomfort and the need for sedation during colonoscopy. Our results indicate the effectiveness of ADover VD in reducing pain and discomfort, improving patient cooperation and willingness to undergo a repeat procedure. To the best of our knowledge, this is the first study to demonstrate the superiority of ADover VD in a head-to-head comparison, showing that AD seems a useful and simple adjunct to low dose sedation during routine colonoscopy.

Our supposition was that VD was likely to be more effective than AD. We theorized that the combination of visual images plus sound would be most engaging and distracting. Previous studies had experimented with the use of a succession of still images, such as lakes, mountains and rivers, and we speculated that film would be even more diverting. The study by Umezawa et al. dem-

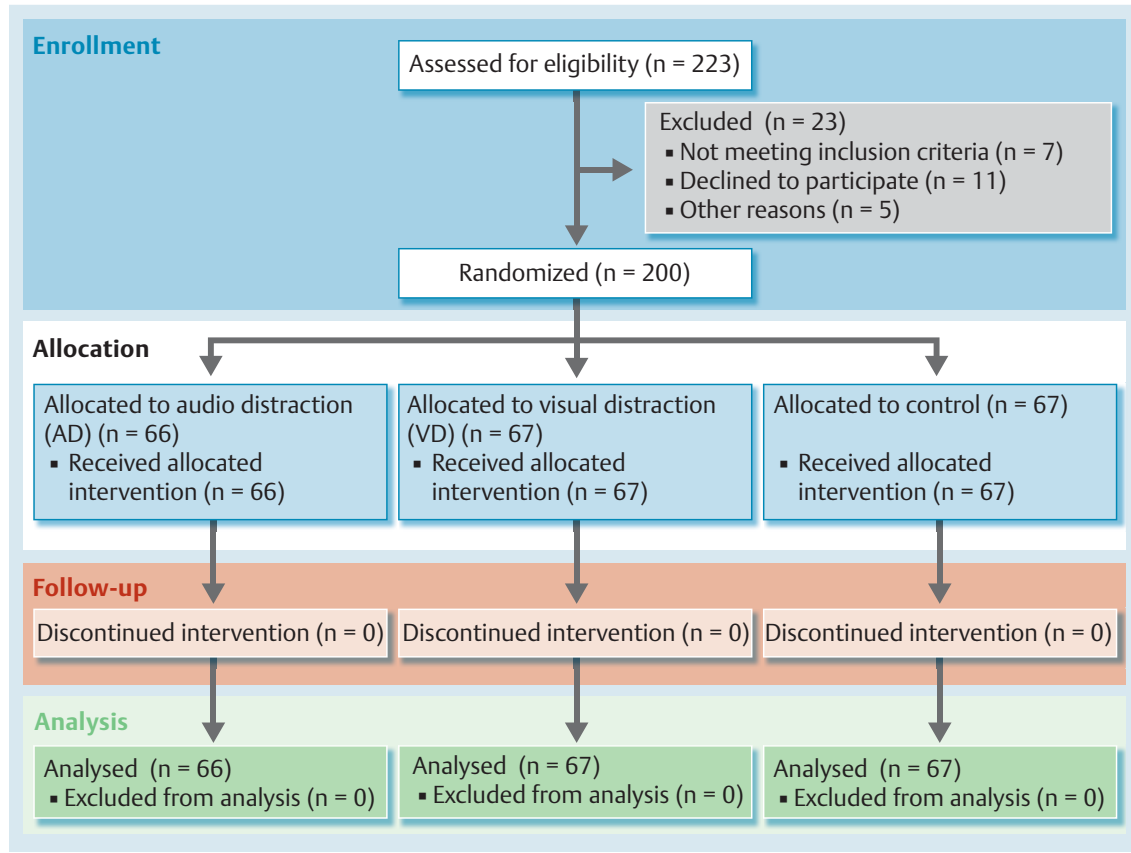

Fig. 2 CONSORT flow chart of study. 
Table 2 Outcomes for the three groups.

\begin{tabular}{|c|c|c|c|c|c|c|c|}
\hline \multirow{3}{*}{$\begin{array}{l}\text { Outcome } \\
\text { Pain score }\end{array}$} & \multicolumn{6}{|c|}{ Group } & \multirow{3}{*}{$\begin{array}{l}P \text { value } \\
<0.001\end{array}$} \\
\hline & \multicolumn{2}{|c|}{ Audio distraction (AD) } & \multicolumn{2}{|c|}{ Visual distraction (VD) } & \multicolumn{2}{|c|}{ Control (C) } & \\
\hline & $3^{1}$ & $(2-4)$ & $4^{2}$ & $(2-6)$ & $5^{2,1}$ & $(3-8)$ & \\
\hline No. of top ups & 1 & $(0-2)$ & 2 & $(1-2)$ & 2 & $(0-3)$ & 0.059 \\
\hline Total dose of pethidine & 10 & $(5-15)$ & 10 & $(10-15)$ & 15 & $(5-20)$ & 0.075 \\
\hline Total dose of midazolam & 1.5 & $(1.0-2.0)$ & 1.5 & $(1.5-2.0)$ & 2 & $(1.0-2.5)$ & 0.087 \\
\hline Willing to repeat colonoscopy & $3^{2}$ & $(3-3)$ & 3 & $(3-3)$ & $3^{2}$ & $(2-3)$ & 0.024 \\
\hline Level of cooperation & $9^{1}$ & $(8-10)$ & $9^{2}$ & $(6-10)$ & $8^{2,1}$ & $(4-9)$ & $<0.001$ \\
\hline
\end{tabular}

Values are median (interquartile range).

$P$ values based on Kruskal-Wallis test.

1 Significant pairwise difference between groups at 0.01 level.

2 Significant pairwise difference between groups at 0.05 level.

onstrated VD using silent movies, to improve patient satisfaction, decrease anxiety and pain during colonoscopy among patients with high pre-procedural anxiety scores [9]. Furthermore, Lembo et al. demonstrated that patients undergoing screening sigmoidoscopies, and receiving a combination of audio and visual interventions had lower discomfort and anxiety levels, compared with no intervention or audio alone [8]. However, the results of the present study showed that the ADgroup listening to their favorite music reported significantly less pain, was more cooperative and was more willing to undergo a repeat procedure than the VD or C groups. The VD group, watching films on a 3D videoglass device, did not achieve the overall pain reduction seen in the ADgroup. Although there was also a trend towards fewer numbers of "top up" doses or total doses of sedation and analgesia used in the ADgroup compared with VD and C groups, this was not statistically significant.

The superiority of AD over VD may be because the VD group was made more alert and awake when watching the film during the procedure which may then have made them more aware of the pain and discomfort of the procedure compared to the AD group. The AD group may have been more "lulled" and distracted by the music which may have augmented the effects of the administered sedation and analgesia.

The masking of randomization might have been ineffective at times in our study. Patients in the VD group watching the movie and the AD group listening to music might in some cases have behaved differently to patients in group C during the procedure. This may have unmasked the randomization to the colonoscopist. We recruited patients presenting for their first colonoscopy as well as those with experience of previous colonoscopies. Although these were only a minority (two in each group), this could have also introduced bias due to the past experience of the exposed patients.

As the sample size we calculated was approximate, during the study our trial statistician recommended an interim analysis after recruiting 200 individuals to make an informed decision as to whether the trial should continue recruiting beyond the planned sample size of 228. As the results of this interim analysis showed unequivocal benefit of AD over VD or sedation alone with regard to most primary outcomes on direct comparison, it was decided to terminate the trial at this stage.
The findings of this study make it practical for patients to bring with them their choice of music on a portable digital music player, to listen during the planned colonoscopy procedure. This will be a simple and effective adjunct to the standard low dose sedation and analgesia for routine colonoscopy. Furthermore, these findings may be applicable to other forms of endoscopic procedures as well. However, further studies are needed to confirm the general applicability of the findings of this study to other areas of endoscopy and to pain management in general.

In conclusion, $\mathrm{AD}$ reduces pain and discomfort, improves patient cooperation and willingness to undergo a repeat procedure, and seems a useful and simple adjunct to standard sedation and analgesia during colonoscopy.

\section{Competing interests: None}

\section{References}

1 Mahajan RJ, Johnson JC, Marshall JB. Predictors of patient cooperation during gastrointestinal endoscopy. J Clin Gastroenterol 1997; 24: 220-223

2 Petravage J, Swedberg J. Patient response to sigmoidoscopy recommendations via mailed reminders. J Fam Pract 1988; 27: 387-389

3 Lee DW, Chan AC, Wong SK et al. Can visual distraction decrease the dose of patient-controlled sedation required during colonoscopy? A prospective randomized controlled trial Endoscopy 2004; 36: 197 201

4 Bechtold ML, Perez RA, Puli SR et al. Effect of music on patients undergoing outpatient colonoscopy. World J Gastroenterol 2006; 12: 73097312

5 Costa A, Montalbano LM, Orlando A et al. Music for colonoscopy: A single-blind randomized controlled trial. Dig Liver Dis 2010; 42: 871 876

6 Smolen $D$, Topp $R$, Singer $L$. The effect of self-selected music during colonoscopy on anxiety, heart rate, and blood pressure. Appl Nurs Res 2002; 15: 126-136

7 Kipping B, Rodger S, Miller K et al. Virtual reality for acute pain reduction in adolescents undergoing burn wound care: a prospective randomized controlled trial. Burns 2012; 38: 650-657

8 Lembo T, Fitzgerald L, Matin K et al. Audio and visual stimulation reduces patient discomfort during screening flexible sigmoidoscopy. Am J Gastroenterol 1998; 93: 1113-1116

9 Umezawa S, Higurashi T, Uchiyama $S$ et al. Visual distraction alone for the improvement of colonoscopy-related pain and satisfaction. World J Gastroenterol 2015; 21: 4707-4714

10 Miller K, Rodger S, Bucolo $S$ et al. Multimodal distraction to relieve pain in children undergoing acute medical procedures [in Chinese]. Zhonghua Shao Shang Za Zhi 2009; 25: 352-356 